\section{Endoscopic placement of a fully covered self-expandable metallic stent to treat an arteriobiliary fistula}

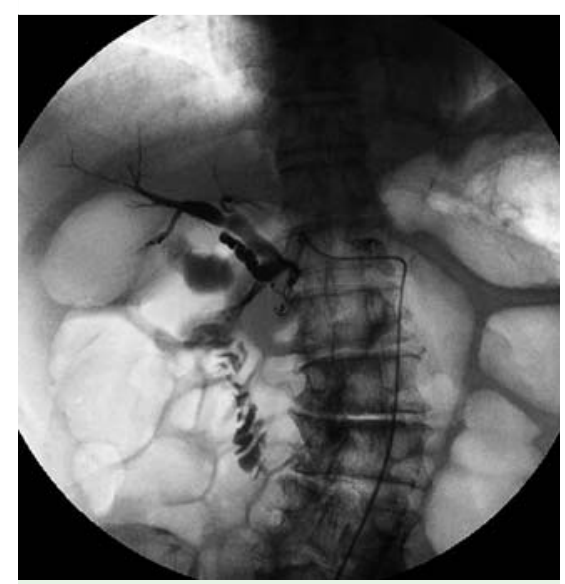

Fig. 1 Angiogram showing an arteriobiliary fistula after contrast injection via the catheterport system for intra-arterial chemotherapy.

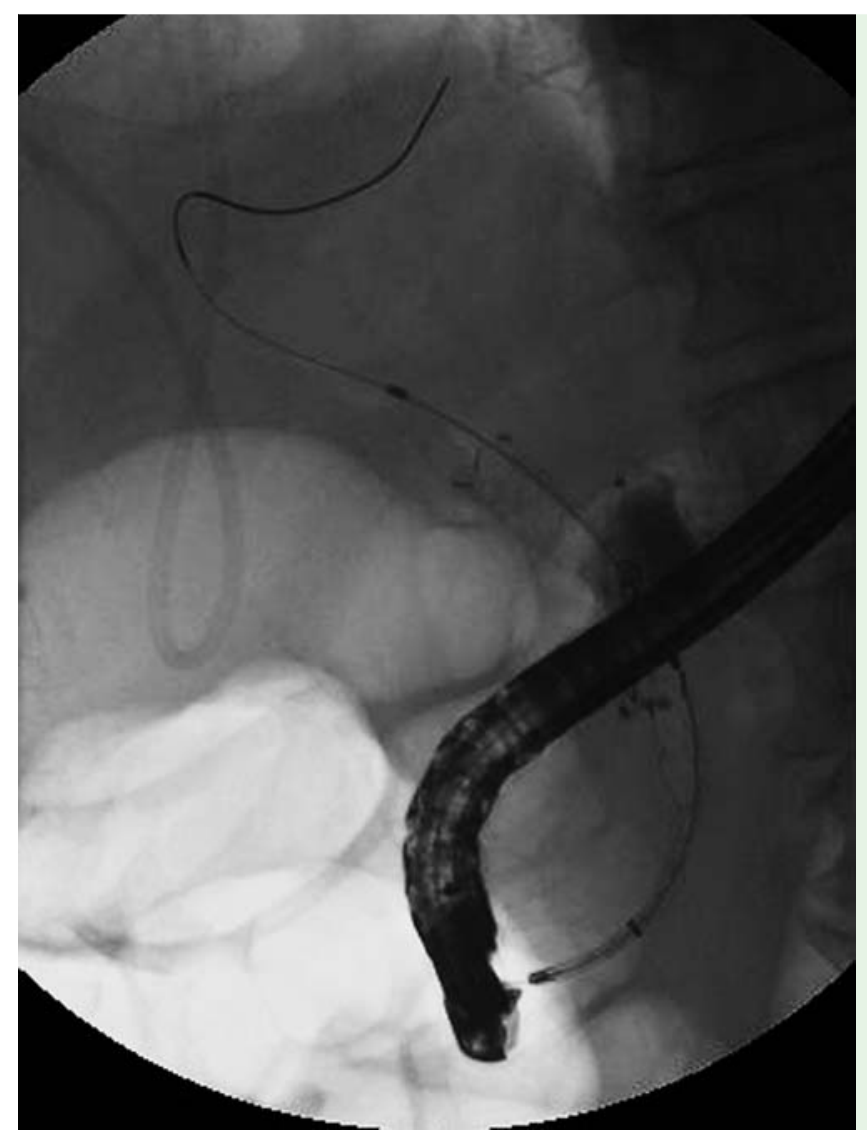

Fig. 3 Radiograph showing the fully covered self-expandable metallic stent placed over a guidewire lying across the arteriobiliary fistula.

Fig. 2 Endoscopic image showing no hemobilia in the ampulla of Vater.
Arteriobiliary fistula is an uncommon complication resulting from hepatic intra-arterial chemotherapy. We present a case of successful endoscopic intervention using a fully covered self-expandable metallic stent (SEMS) to treat an iatrogenic arteriobiliary fistula.

A 71-year-old man with multiple liver metastases from pancreatic cancer was admitted. He had undergone distal pan- createctomy. He had also undergone chemotherapy with multiple regimens during the past 43 months and percutaneous transfemoral implantation of the catheter-port system for intra-arterial chemotherapy to treat his liver metastases. Three months after catheter placement, he developed sepsis, with positive results on bacterial blood cultures. The reason for the sepsis could not be determined using computed tomography. However, angiography via the catheter revealed a fistula between the hepatic artery and the extrahepatic bile duct ( $\bullet$ Fig. 1 ), indicating the fistula as the cause of the sepsis. Because of catheter-related thrombosis, the implanted catheter-port system could not be removed, so we decided to seal the arteriobiliary fistula endoscopically. Duodenoscopy revealed no hemobilia in the ampulla of Vater ( $\bullet$ Fig. 2). A fully covered SEMS (ComVi, 10×80 mm; Taewoong-Medical Co., Ltd., Gyeonggi-do, Korea) ( $\bullet$ Fig.3) was inserted, and the fistula was sealed successfully without any complications ( $\bullet$ Fig.4). The patient did not require any treatment for the arteriobiliary fistula thereafter.

Arteriobiliary fistula after intra-arterial chemotherapy has been reported in only a few cases [1-4]. Embolization of the catheter track or the branch of the hepatic artery is a reported treatment [4]. More recently, percutaneous transhepatic placement of a covered SEMS to treat an intrahepatic arteriobiliary fistula has been described [5]. To our knowledge, however, ours is the first report of an arteriobiliary fistula treated endoscopically using a fully covered SEMS. Endoscopic placement of a fully covered SEMS should be recognized as a treatment option for arteriobiliary fistulas, as it could obviate the need for an arteriogram.

Endoscopy_UCTN_Code_TTT_1AR_2AF

\section{Competing interests: None}

\section{Hiroshi Kawakami ${ }^{1}$, Munenori Okamoto $^{2}$, Masaki Kuwatani ${ }^{1}$, Yoshimasa Kubota', Kazumichi Kawakubo', Yoko Abe', Shuhei Kawahata', Kimitoshi Kubo ${ }^{1}$, Naoya Sakamoto ${ }^{1}$}

\footnotetext{
${ }^{1}$ Department of Gastroenterology and Hepatology, Hokkaido University Graduate School of Medicine, Sapporo, Japan

2 Department of Internal Medicine, Sapporo Century Hospital, Sapporo, Japan
} 


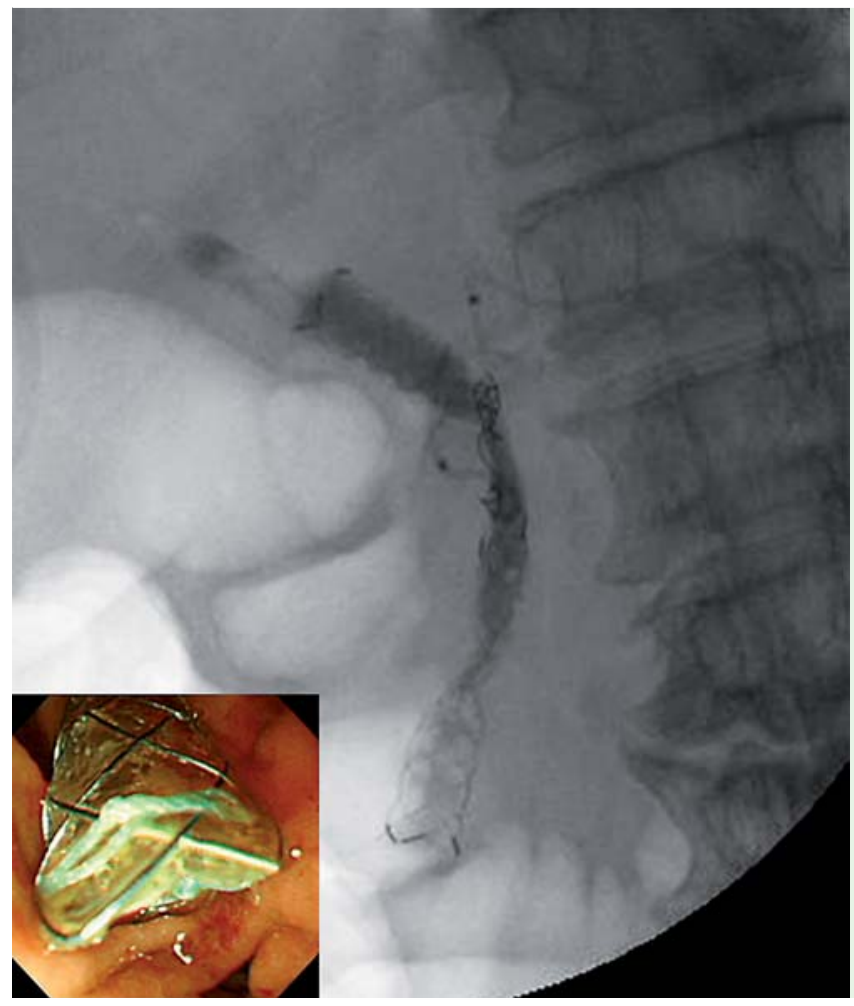

Fig. 4 Radiograph showing the arteriobiliary fistula successfully sealed using the fully covered self-expandable metallic stent (inset: endoscopic view of the stent passing through the ampulla of Vater to the duodenum).

\section{References}

1 Rosenberg HD, Wile AG, Aufrichtig D et al. Hepatic artery-biliary fistula. An unusual complication of infusion therapy. Gastrointest Radiol 1983; 8: 37-40

2 Noda M, Kusunoki M, Yanagai $H$ et al. Hepatic artery-biliary fistula during infusion chemotherapy. Hepatogastroenterology 1996; 43: 1387-1389

3 Venturini M, Angeli E, Salvioni M et al. Complications after percutaneous transaxillary implantation of a catheter for intraarterial chemotherapy of liver tumors: Clinical relevance and management in 204 patients. Am J Roentgenol 2004; 182: 1417-1426

4 Takao H, Doi I, Makita K et al. Coil embolization of an arteriobiliary fistula caused by hepatic intra-arterial chemotherapy. Cardiovasc Intervent Radiol 2005; 28: 825-827

5 Tan KC, Kapoor BS. Hepatic arteriobiliary fistula successfully treated with an endobiliary covered stent. J Vasc Interv Radiol 2008; 19: $1521-1522$

\section{Bibliography}

DOI http://dx.doi.org/

10.1055/s-0034-1377939

Endoscopy 2014; 46: E566-E567

(C) Georg Thieme Verlag KG

Stuttgart · New York

ISSN 0013-726X

\section{Corresponding author}

\section{Hiroshi Kawakami, MD, PhD}

Department of Gastroenterology and Hepatology Hokkaido University Graduate School of Medicine Kita 15, Nishi 7, Kita-ku Sapporo 060-8638

Japan

Fax: +81-11-7067867

hiropon@med.hokudai.ac.jp 